\title{
Mekuido: intervención en línea para escolares chilenos de apoyo a la prevención del sobrepeso
}

\author{
Mekuido: on line intervention for chilean school \\ children to support prevention of overweight
}

\begin{abstract}
The MeKuido intervention is described, designed for the project "Prevention of overweight and obesity in Chilean children: Bettinhg on the enterprising capacity of children" (FONIS SA10120016). Subjects and Method: A descriptive intervention study on a randomly selected population by cluster at $50 \%$ of municipal schools in the district of La Pintana, with children enrolled in 5th and 6th grade of primary school who agreed to participate $(n=468)$. Mekuido, central element is a software with four sections built according to Orem's referential framework. Post intervention, a satisfaction questionnaire was applied. Results: The implementation in the context of school activities proved feasible but not without difficulties. The satisfaction questionnaire was answered by 355 children (90.8\%). All closed questions showed over $80 \%$ of satisfaction with the intervention and would recommend their use. Conclusion: The Orem framework allows the design of interventions to change behaviors related to feeding. Its use is feasible in the school context, requires determination and commitment of teachers. The strategy can be incorporated into the curriculum and contribute to the development of transversal objectives.

Key words: Child nutrition; overweight; obesity; self care; feeding behavior.
\end{abstract}

\author{
M. Cecilia Campos S. (1) \\ Sonia Jaimovich P. (1) \\ María Sylvia Campos S. (1) \\ Luz María Herrera L. (1) \\ Verónica Lillo S. (2) \\ Dayann Martínez S. (1) \\ María Paz Del Río C. (1) \\ Valentina Arellano F. (1)
}

(1) Escuela de Enfermería, Pontificia Universidad Católica de Chile. Santiago, Chile (2) Facultad de Educación, Pontificia Universidad Católica de Chile. Santiago, Chile.

$$
\begin{array}{r}
\text { Dirigir la correspondencia a: } \\
\text { Profesora } \\
\text { María Cecilia Campos S. } \\
\text { Escuela de Enfermería } \\
\text { Pontificia Universidad Católica de Chile } \\
\text { Vicuña Mackenna 4860, Macul, Santiago de Chile } \\
\text { Fono: 23545839 } \\
\text { Fax: 23547025 } \\
\text { E-mail: ccampos@uc.cl. }
\end{array}
$$

Este trabajo fue recibido el 11 de Septiembre de 2012 y aceptado para ser publicado el 20 de Enero de 2013.

\section{INTRODUCCIÓN}

La malnutrición por exceso continúa aumentando significativamente, es un problema de salud pública creciente en todo el mundo, incluido los países de Latinoamérica. Esta situación ha llevado a la OMS a declarar la obesidad, epidemia del siglo.

En Chile las últimas cifras entregadas por la Encuesta Nacional de Salud 2009-2010, muestran que un 39.3\% de la población mayor de 15 años presenta sobrepeso, 25.1\% obesidad y $2.3 \%$ obesidad mórbida, siendo mayor el problema en hombres que en mujeres en todos los grupos de edad. Esto difiere de la percepción de las personas, quienes al pedirle que se identifiquen con los diferentes conceptos, en un $42.6 \%$ se catalogan como normales, $46.3 \%$ como sobrepeso y sólo un $3.8 \%$ como obesos (1). En los niños/as chilenos para 2007, el $9.6 \%$ de los menores de 6 años y 19.4\% de escolares de $1^{\circ}$ básico presentan obesidad $(2,3)$. En Chile, los/as niños/as que ingresan al sistema público de educación son los/as que presentan las tasas más altas de obesidad (4).

La diferencia entre lo percibido y lo real dificulta el cambio de conducta debido a la falta de conciencia de las personas que presentan el problema. Esta diferencia justifica, entre otros, la necesidad de que los niños/as desde edades tempranas tengan conocimiento claro de cuál es su estado nutricional y las posibilidades de modificar los factores que influyen en él.

En la pre adolescencia los niños/as comienzan a decidir sobre los alimentos que consumen y a desarrollar los patrones alimentarios que se traducirán en comportamientos futuros. (5) Estudios del País Vasco han demostrado que antes de los 16 años aún es factible modificar los hábitos, es la edad en que se opta por actividades sanas o cuando surgen los patrones de conducta de riesgo (6).

A partir de estos antecedentes y con el propósito de contribuir al logro de objetivos sanitarios en nuestra población, se diseñó una propuesta interdisciplinaria desde Salud y Educación, de apoyo a la prevención del sobrepeso y la obesidad en escolares chilenos. Esta intervención consistió en la aplicación de la estrategia denominada "MeKuido" en niños/as escolares de $5^{\circ}$ y $6^{\circ}$ año de educación general básica, de colegios municipalizados de la comuna La Pintana, Región 
Metropolitana de Chile; y se orientó al cambio positivo de conductas de autocuidado asociadas a la alimentación y su correspondiente correlato a las prácticas familiares (7).

El término "MeKuido" es un constructo co creado por el equipo de investigadores con la participación de representantes de la comunidad a la que está dirigida la intervención. Hace referencia, de manera general, al autocuidado de la salud $y$, de manera particular, al desarrollo de la autoresponsabilidad y la participación activa en el cuidado del estado nutricional, a través de la toma de decisiones informada sobre las mejores prácticas de autocuidado relacionadas con una alimentación saludable que permitan mantener un peso óptimo. Por esto último la letra $\mathrm{K}$ (de kilo) al centro de la palabra.

Este artículo, como parte del proyecto "Prevención del sobrepeso y de la obesidad en escolares chilenos: Una apuesta a la capacidad emprendedora de los niños" (FONIS SA10120016), describe la intervención MeKuido, el proceso de implementación en el contexto de las actividades escolares y la satisfacción de los estudiantes con el uso del software. No se reportan los resultados del efecto de la intervención en términos de cambio en las conductas alimentarias de los niños/as.

Continúa una línea de trabajo de los autores relacionada con la prevención de sobrepeso y obesidad en escolares.

Cabe señalar que este estudio, en el contexto de la responsabilidad social, se ha desarrollado en una de las comunas de la Región Metropolitana con más altos índices de vulnerabilidad social, según el indicador de calidad de vida urbana (8).

\section{SUJETOS Y MÉTODO Diseño}

Estudio descriptivo correspondiente al proceso de implementación de la estrategia MeKuido en estudiantes del grupo intervenido del proyecto FONIS SA10I20016 durante el período de junio a noviembre de 2011 y a la satisfacción de los estudiantes del grupo intervenido. Este proyecto fue aprobado por el Comité de Ética de la Escuela de Enfermería de la Pontificia Universidad Católica de Chile.

\section{Sujetos}

Mediante un muestreo aleatorio por conglomerado, se seleccionó al 50\% de los colegios municipalizados de la comuna de La Pintana, donde cada colegio se constituyó como unidad de aleatorización. La población estuvo conformada por todos los niños/as que en marzo del año 2011 cursaban $5^{\circ}$ y $6^{\circ}$ año básico, en 6 colegios municipalizados, quienes formaron el grupo intervenido del proyecto $(n=468)$. Tanto los niños/as como sus padres o apoderados, firmaron un consentimiento informado para aceptar y comprometer su participación. Se obtuvo la autorización correspondiente de las autoridades educacionales a nivel de municipio y de dirección del establecimiento.

\section{Estrategia de intervención MeKuido}

MeKuido es una estrategia tecnológica simple y autosustentable, construida con apoyo del marco de referencia de la teoría de Déficit de Autocuidado de D. Orem. Esta teoría destaca que el autocuidado es un fenómeno activo que requiere que las personas sean capaces de usar la razón para comprender su estado de salud y decidir un curso de acciones apropiadas en beneficio de su salud y bienestar, condicionado por los conocimientos y repertorio de habilidades de las persona. Uno de los supuestos de la teoría es que el autocuidado se aprende en el proceso de crecimiento y desarrollo (9).

La estrategia fue diseñada para empoderar a niños/as de $5^{\circ}$ a $7^{\circ}$ básico para que asuman el desafío de modificar sus conductas alimentarias en pro de su buen estado nutricional, de su salud en general y la de su familia. Puede contribuir al logro de objetivos educativos transversales tales como el desarrollo de autoestima positiva, motivación, voluntad y habilidades sociales.

El elemento central de la estrategia MeKuido fue el software MeKuido.info (http://www.mekuido.info/), aplicación Web, que ofreció al niño(a) actividades para comprender y evaluar sus prácticas de autocuidado asociadas a la alimentación y combinar sus habilidades personales con la creación de planes y propuestas de cambio de conductas. El software presentó al alumno/a cuatro secciones en las que debió trabajar semanalmente en forma personal en la hora pedagógica que el colegio destinó a la actividad. El trabajo personal fue supervisado y apoyado por un profesor interiorizado y comprometido con este programa. En visitas de supervisión del proceso de intervención realizadas quincenalmente, se entregó a los profesores participantes información y retroalimentación en relación al funcionamiento y mejor uso del software y se estimuló la participación y el mejor uso del tiempo.

La primera vez que el niño ingresó al software, además de digitar sus datos de identificación, peso y talla, eligió una figura "avatar", que desde ese momento en adelante lo acompañó durante el uso de este medio.

En la primera sección "Que comí", los niños/as ingresaban los alimentos consumidos el día anterior: (recordatorio de 24 hrs). Con estos datos el software le entregaba como resultado las calorías consumidas, la distribución según macronutrientes, su relación con su estado nutritivo y una recomendación al respecto.

En la segunda sección "Trivia", construida según el marco de referencia de modelo de Déficit de Autocuidado de Orem (9), el programa presentaba al alumno/a en forma aleatoria, una serie de preguntas asociadas a las prácticas para ser respondidas cada semana. Ellas fueron elaboradas con el fin de estimular en el niño/a una reflexión para comprender y analizar sus prácticas o hábitos alimentarios, valorar y reconocer su responsabilidad en el cuidado de su nutrición. A continuación el software entregaba al niño/a un comentario para cada una de sus respuestas, un mensaje con contenido educativo y una frase o pregunta para la reflexión.

La Trivia contempló 3 categorías de preguntas:

- de búsqueda de información y conocimientos, para evaluar si los niños/as están preocupados de buscar información, conocer del tema, discutirlo con alguien, así como conocer cuáles son sus principales fuentes de información, a quienes y dónde consultan.

- de elecciones y toma de decisiones, para evaluar si los niños/ as realizan elecciones y toman decisiones relacionadas con su alimentación y

- de acciones concretas, para saber que están haciendo los niños/as en relación al cuidado de su alimentación y nutrición.

Las preguntas de la Trivia abordaron conocimientos básicos de nutrición, alimentos, sustancias nutritivas, estado nutricional, pirámide nutricional, alimentación saludable, composición de una dieta balanceada, selección, conservación y preparación de alimentos, alimentación en el ciclo de vida, información nutricional, publicidad de los alimentos, efectos de una mala alimentación, relación entre alimentación y actividad física, proyectos de alimentación saludable en la escuela, enfermedades asociadas a la alimentación; a la malnutrición: sobre peso y obesidad. Algunas de estas temáticas están 
incluidas en Chile en los planes de estudio de $5^{\circ}$ y $6^{\circ}$ básico. En la tercera sección "Mis compromisos", los niños/as escribían propuestas para mejorar sus prácticas, las que deberían revisar en la próxima sesión para evaluar su cumplimiento. El profesor/a debía analizar con ellos los compromisos y reforzar positivamente al trabajo realizado; de esta manera actuó como referente y líder en el fortalecimiento y consolidación de conductas saludables en los alumnos/as.

La última sección del software "Ayudas" ofreció a los niños/as el siguiente material de apoyo: a) mensajes Mekuido que son consejos para conocer más del tema, para elegir y tomar buenas decisiones y para mejorar las prácticas en alimentación; b) un glosario con términos generales referido a los principales conceptos usados en el software; c) un cuadro de alimentos saludables con ideas para confeccionar una minuta diaria y d) la pirámide de actividad física para niños/as.

La intervención se diseñó para que el niño/a trabaje con cada una de las secciones del software semanalmente durante 3 semanas; la semana $n^{\circ} 4$ era una sesión grupal dedicada a poner en común el trabajo realizado, socializar sus compromisos y recibir feedback o retroalimentación por parte de su profesor/a y sus pares. Este ciclo de 3 semanas de trabajo personal más una semana de trabajo grupal, se debía repetir durante 15 semanas o 4 meses (figura 1).

Finalmente y a modo de cierre y evaluación formativa del proceso completo, se realizó una jornada formativarecreativa que se denominó: "Olimpíadas MeKuido: ¿Quién sabe más?"; la que contempló una serie de competencias de destreza física y la aplicación de los conocimientos adquiridos a través del software. Participaron todos los colegios del grupo experimental.
El desarrollo de la intervención MeKuido, consideró la recalificación de los profesores previa a su inicio y un sistema de supervisión permanente a cargo del equipo de investigación. La supervisión realizada mediante visitas quincenales a los colegios, consistió en observar el ejercicio en terreno y realizar una entrevista personal a la profesora o profesor a cargo. Complementariamente a través del sitio web, se realizó un seguimiento del número de visitas y de las actividades realizadas por los estudiantes en el software.

Se aplicó una encuesta opinión sobre el uso del software MeKuido a 391 escolares y de ellos 355 contestaron la encuesta (90,8\%). 26 alumnos/as respondieron que nunca lo usaron y 10 la entregaron en blanco. Este cuestionario de satisfacción contenía 11 preguntas, de las cuales 6 eran cerradas, con alternativa de respuesta si/no y 5 abiertas.

\section{RESULTADOS}

Proceso de implementación MeKuido en el contexto de las actividades escolares

El proceso de implementación se diseñó para trabajar con los niños/as de 5 y $6^{\circ}$ básico de 6 colegios municipalizados de la comuna de La Pintana Región Metropolitana. Un colegio fue eliminado de la muestra por encontrarse en paro estudiantil durante el período de la intervención (n 26), por lo que el grupo estudiado quedó conformado por 5 colegios y 442 niños/as (tabla 1).

Después de obtenido el consentimiento informado de los padres o apoderados de los niños/as y la aceptación de participación de ellos, se inició la intervención la primera semana del mes de junio del año 2011. Cada colegio designó un profesor y una hora semanal para el trabajo de los niños/as

\section{FIGURA 1}

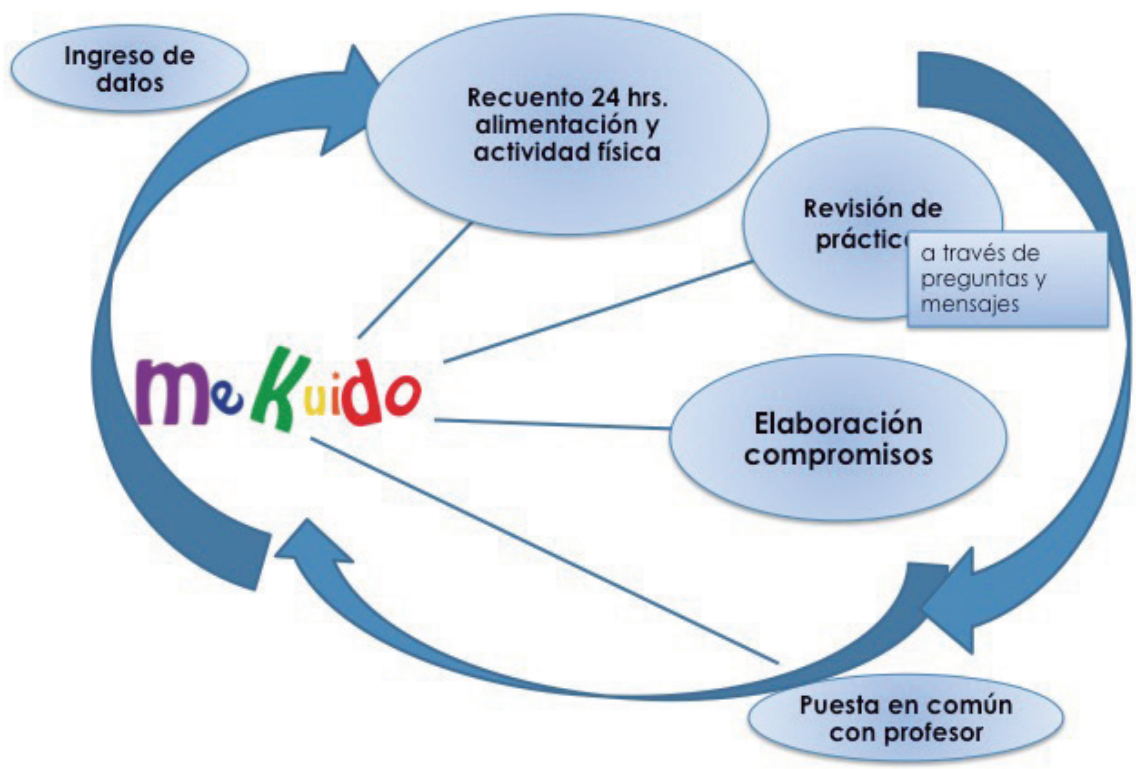


por un período de 15 semanas: 3 semanas de trabajo personal en sala de computación y una semana de trabajo grupal en sala de clases.

Una vez cumplidas las 15 semanas se puso fin a la intervención con la jornada formativa-recreativa "Olimpíadas MeKuido : ¿Quién sabe más?" en la que participaron niños/as de todos los colegios del grupo en estudio. En ella, a través de competencias, los niños/as respondían preguntas vinculadas al uso de MeKuido, demostrando con ello lo aprendido.

De apoyo al trabajo de los profesores se puso a su disposición el "Manual del profesor" que contiene una explicación detallada del proceso y algunos fundamentos teóricos referidos al autocuidado, y al proceso de cambio de conducta y el tríptico "MeKuido: guía de referencia rápida" que contiene las instrucciones para trabajar con el software.

Con el propósito de asegurar condiciones de éxito en la experiencia, se recalificó previamente al cuerpo de profesores, explicitando los objetivos, el funcionamiento de la intervención MeKuido, el diseño metodológico de las actividades y el sistema de seguimiento y evaluación formativa del proceso. Particularmente, se acentuó la necesaria adscripción del compromiso de los profesores y las altas expectativas con respecto a su desempeño. Es importante destacar que en esta primera actividad con los/as profesores/as se constató, en la mayoría de los casos, una barrera para el éxito del estudio, la que se denominó "el factor dirección". Los equipos directivos de los establecimientos, pese a su compromiso previo en la adscripción al proyecto, no facilitaron las condiciones requeridas para el buen desempeño de los profesores/as y sus estudiantes (asignación de tiempos, y recursos materiales).

El ingeniero encargado del desarrollo de software, comprobó las condiciones de las salas de computación de los colegios de modo de asegurar su funcionamiento.

La supervención de la intervención que estuvo a cargo de los investigadores mostró que: en promedio, cada establecimiento educacional contaba con 23 computadores en la sala de computación, con conexión a internet y velocidad adecuada a excepción de un colegio con baja velocidad; en todos los casos, los estudiantes se sentaron en pareja frente al computador y se turnaban para trabajar individualmente en su ejercicio y que el promedio de tiempo por sesión de cada niño/a fue de 20 minutos.

Todos los profesores supervisaron a sus estudiantes para evitar que no entraran en otros programas, pero sólo algunos (menos del 50\%) monitorearon el trabajo de los estudiantes y respondieron sus consultas. No todos los colegios cumplieron con el calendario de sesiones establecidas y en general no se recuperaron las sesiones perdidas por falta de tiempo para realizarla.

Según apreciación de los profesores/as el nivel de participación de los estudiantes evaluado en escala de 0 a 10, osciló entre los puntajes 5 y 9,5. Se aprecia una tendencia a percibir mejor participación en las sesiones posteriores, en la medida que los niños/as adquieren más experiencia. Esta percepción es mayor que la participación de los estudiantes, medida a través del software. En relación a las sesiones de trabajo grupal, en tres de los 5 colegios se realizó en la sala de clase y en el resto en la sala de computación. El promedio por sesión grupal fue de 40 minutos, fluctuando desde 5 a 90 minutos. Esta situación es la que presenta mayor diferencia entre los distintos establecimientos educacionales.

La estadística aportada Google Analytics, mostró que durante el periodo que duró la intervención (15 semanas), el número de visitas al sitio MeKuido.info fue de 2608, de las cuales $83.36 \%$ se pueden atribuir a los estudiantes que sistemáticamente usaron el sitio; el porcentaje restante corresponde a visitas realizadas por una sola vez. En relación a la duración de la sesión, en un 43,7\% fue de más de 10 minutos, seguida de un $28 \%$ entre 3 y 10 minutos. La sección más visitada fue la "Trivia" con un 35,7\% del total de visitas, le siguen en orden de frecuencia "Que comí" y Mis compromisos".

La sección "Mis compromisos" si bien fue visitada, no cumplió con el objetivo, ya que los compromisos elaborados por los niños/as fueron insuficientes en cantidad y calidad.

En relación al número de veces que los niños/as cumplieron con el calendario establecido para el trabajo personal, sólo 1 de cada 3 niños/as cumplió con el trabajo individual durante 8 y más semanas (94 de 300).

Satisfacción con el uso del software MeKuido

Para conocer la satisfacción de los estudiantes con el uso del software MeKuido se aplicó una encuesta de 11 preguntas (6 cerradas y 5 abiertas) a 391 escolares, con una tasa de respuestas de $90,8 \%$ (355). Más del $80 \%$ de los estudiantes respondió que le gustó trabajar con este software, que fue entretenido, fácil y que lo recomendaría a un amigo. El 64, $8 \%$ comentó en su casa lo aprendido con MeKuido (tabla 2).

Para aproximadamente la mitad de los estudiantes (186) lo que "más les gustó" fue alguna de las actividades del software, entre las que se destaca la Trivia (108); para alrededor

\section{TABLA 1}

Distribución de niños según colegios pre y post intervención Abril y Noviembre 2011

\begin{tabular}{lccc} 
Colegios & $\begin{array}{c}\text { Pre intervención } \\
\text { abril } 2011\end{array}$ & $\begin{array}{c}\text { Post intervención } \\
\text { noviembre 2011 }\end{array}$ & $\begin{array}{c}\text { Sin aplicar } \\
\text { retirados/ausentes }\end{array}$ \\
Centro Educacional La Pintana & 95 & 80 & 15 \\
Víctor Jara & 104 & 92 & 12 \\
Pablo de Rokha & 71 & 66 & 5 \\
Neruda & 56 & 53 & 3 \\
Aurelia Rojas & 116 & 88 & 28 \\
Mariano Latorre* $^{*}$ & 26 & 0 & 26 \\
\hline
\end{tabular}

* Colegio eliminado de grupo intervención 
de un tercio (117) fue aprender a cuidar su salud y tener una alimentación saludable, lo que se refleja en respuestas como: "me enseñó a comer mejor", "aprendimos a cuidar nuestra salud", "aprender a elegir los alimentos"; y para el resto fueron aspectos no relacionados con el uso del software, tales como las olimpiadas, salir de clases, conectarse a internet, jugar y divertirse, compartir o trabajar en equipo.

Alrededor del 25\% de los estudiantes mencionan que lo que "menos les gustó", fueron los problemas de conexión o de funcionamiento del software y el tiempo destinado a la actividad, lo que se expresa en comentarios como: "ir todos los días", "perder horas de clases", "no fue fácil trabajar", "dura muy poco rato", "que no salían los alimentos que uno comía", "eran muchas cosas y uno se demoraba".

Respecto del principal o más importante aprendizaje obtenido con el uso del software, las principales respuestas se relacionan con: aprender a cuidar la salud y tener una alimentación saludable (251), lo que se manifiesta en comentarios como "comer sano o saludable", "cuidarse o cuidar de la salud", "no se debe comer lo que no es saludable" "lo que es bueno comer"; y con aprender las características de los alimentos, macronutrientes, cuales son los alimentos saludables, la pirámide alimentaria o leer las etiquetas de alimentos (44).

Por último, entre las palabras más mencionadas por los estudiantes para calificar su experiencia con el uso del software MeKuido se encuentran: "aprendizaje", "muy bueno", "bacán", "excelente", "entretenido", "lindo", "simpático", "alegre", "amoroso", "amigable", "interesante", "importante", "buen espacio", "felicidad", "saludable", "gracias". Un bajo número de estudiantes (12) calificó la experiencia como "fome o aburrida".

\section{DISCUSIÓN Y CONCLUSIONES}

Los resultados de este estudio demuestran una vez más la factibilidad de realizar intervenciones destinadas a prevenir la malnutrición por exceso en población de escolares, como las realizadas en Chile por Kain et al. y Vío et al. $(10,11)$. La innovación de la intervención comunicada radica en que, en este caso se focaliza en la elaboración de propuestas de cambio de conductas alimentarias realizadas por los propios niños/as.

El cambio de conducta es un componente fundamental para lograr cualquier respuesta frente al sobrepeso y a la obesidad, sin embargo, es un proceso complejo que va más allá que la sola educación escolar y entrega de información. Se hacen necesarios cambios en el ambiente para prevenir conductas poco saludables. La evidencia sugiere que las estrategias actualmente utilizadas están fallando en la obtención de impacto suficiente porque no cubren todas las variables que influyen $(4,12,13)$.

El marco de referencia de Orem con el cual se diseñó la intervención para motivar el cambio de conductas asociadas a alimentación, es adecuado y coherente con el instrumento Moore Index of Nutrición Child (MIN-C(C) con los cuales se midió el cambio. Ha sido utilizado en investigaciones realizadas por Moore J.B., et al $(14,15)$ y fue validado y utilizado en población de escolares chilenos por el grupo que lidera el proyecto Fonis $(16,17)$.

El uso de la estrategia MeKuido en el contexto escolar, es factible, requiere de decisión y compromiso de los equipos directivos y profesores. La estrategia puede ser incorporada al curriculum escolar y contribuir al desarrollo de objetivos transversales como crecimiento y autoafirmación personal orientados al desarrollo de la identidad personal, la autoestima, la afectividad y la capacidad de formular proyectos de vida; desarrollo del pensamiento orientados a la promoción de las habilidades para desarrollar ideas y conocimientos, analizar información y resolver problemas; manejo de Tecnologías de Información y Comunicación (TICS) orientados a promover un uso eficiente y responsable de las TICs, potenciando el aprendizaje y desarrollo personal $(18,19)$. Para ello es fundamental que el profesor oriente, facilite y supervise el trabajo de cada niño, entregue refuerzo positivo o retroalimentación, fomente la iniciativa y el trabajo autónomo. Pocos profesores monitorearon el trabajo de los estudiantes, respondieron sus consultas y apoyaron la formulación de compromisos. El estudio exploratorio realizado por un equipo del Instituto de Informática Educativa de la universidad de La Frontera (20) evidencia que las Tics por sí misma no tienen efecto sino que están asociadas a la práctica docente.

Solo 1 de cada 3 niños/as cumplió con más del 60\% de las sesiones individuales programadas según la estadística aportada por Google Analytics. Esto muestra una insuficiente adherencia que afecta en forma negativa el impacto del programa. La percepción que los profesores/as reportaron de la participación de los estudiantes es que ésta es mayor que la medida a través del software. El "factor dirección" y "factor profesor/a" se constituyeron en barreras para el cumplimento de la intervención tal como fue diseñada.

Los equipos directivos de los establecimientos no establecieron las condiciones requeridas para la asignación de los tiempos, realización de las actividades y adjudicación de las

TABLA 2

Satisfacción de los niños/as con el software MeKuido

(N 355) Noviembre 2011

\begin{tabular}{|c|c|c|c|c|}
\hline \multirow[t]{2}{*}{ Preguntas } & \multicolumn{2}{|c|}{$\mathrm{Si}$} & \multicolumn{2}{|c|}{ No } \\
\hline & $\mathrm{N}^{\circ}$ & $\%$ & $\mathrm{~N}^{\circ}$ & $\%$ \\
\hline ¿Fue fácil trabajar MeKuido? & 299 & 84,2 & 26 & 7.3 \\
\hline ¿Aprendiste cosas nuevas con MeKuido? & 328 & 92,4 & 14 & 3,9 \\
\hline ¿Fue entretenido trabajar con MeKuido? & 320 & 90,1 & 18 & 5,1 \\
\hline ¿Te gustó trabajar con MeKuido? & 314 & 88,5 & 21 & 5,9 \\
\hline ¿Lo recomendarías a un amigo? & 285 & 80,3 & 42 & 11.8 \\
\hline ¿Has comentado en tu casa lo aprendido con MeKuido? & 230 & 64,8 & 98 & 27.6 \\
\hline
\end{tabular}


responsabilidades de sus profesores. La supervisión por parte de los profesores fue escasa, con una tendencia a atribuir a los estudiantes la autorregulación de su conducta para desempeñarse en el software. A pesar de las condiciones iniciales desde las cuales trabajaron los/as profesores/as, éstos mostraron un particular compromiso con el proyecto y una gran capacidad de persistencia para finalizar el proceso.

Para el éxito de intervenciones a nivel escolar es indispensable que estas sean parte de las actividades escolares programadas y con tiempo protegido. Un estudio piloto desarrollado para determinar el impacto del Modelo de Curriculum de Nutrición de Michigan, expectativas de eficacia y comportamientos de alimentación en estudiantes con promedio de edad de 12 años, comprobó la necesidad que las intervenciones sean parte de un curriculum para obtener resultados. En este estudio se preparó a profesores/as, quienes enseñaron a los estudiantes del grupo intervenido. Los resultados mostraron que los niños/as mejoraban sus conocimientos post intervención y que este grupo más adelante, tenía mayor disposición hacia el consumo de frutas y verduras y menor disposición hacia la comida chatarra en comparación con el grupo control, así como mayor confianza en consumir una alimentación saludable (21).

En este contexto, cabe hacer mención a la necesaria fidelización de los profesores con respecto al estudio, tal como se ha señalado en otras investigaciones $(22,23)$. En este caso, pese al proceso de recalificación de los profesores para la aplicación de la intervención MeKuido, éstos no mostraron un adecuado nivel de adhesión, en ninguno de los tres aspectos de la fidelidad de la implementación (aplicación, cumplimiento y calidad de la entrega); consecuentemente este factor se constituye en una variable que debe ser regulada eficientemente en posteriores intervenciones. Sin embargo, cabe señalar que a pesar de las condiciones adversas desde las cuales trabajaron, los/as profesores/as mostraron gran capacidad de persistencia para finalizar el proceso.

Se comprueba que la implementación de una estrategia de este tipo, fue factible sin grandes problemas, con recursos de los establecimientos municipalizados de la comuna de La Pintana y que la satisfacción reportada por los niños/as con la intervención fue alta. Respecto de los resultados de la encuesta de satisfacción, preocupa el hecho que solo el $64,8 \%$ compartiera su experiencia con su familia. Esto sugiere abordar intencionadamente la interacción del escolar con su familia mediante acciones que la estimulen.

En Chile, al igual que en otros países han surgido políticas gubernamentales que promocionan e incentivan estrategias destinadas a mejorar los estilos de vida; el Programa Elige Vivir Sano, iniciativa del Gobierno de Chile implementada el año 2011, es un ejemplo de ello. Este programa propone a los ciudadanos cuatro compromisos para mejorar su calidad de vida los cuales son: alimentación saludable, actividad física, vida en familia y vida al aire libre (24).

\section{RESUMEN}

Se describe la intervención MeKuido diseñada para el proyecto "Prevención del sobrepeso y de la obesidad en escolares chilenos: Apuesta a la capacidad emprendedora de los niños" (FONIS SA10I20016). Sujetos y Método: Estudio descriptivo de la intervención realizada a población seleccionada mediante muestreo aleatorio por conglomerado del 50\% de los colegios municipalizados de la comuna de La Pintana, conformada por los niños/as que en marzo de 2011, cursaban $5^{\circ}$ y $6^{\circ}$ año básico, $(n=468)$ y aceptaron participar. Mekuido elemento central de la intervención, es un software con cuatro secciones construidas según marco referencial Orem. Post intervención, se aplicó cuestionario de satisfacción. Resultados: La implementación en el contexto de las actividades escolares demostró ser factible, pero no exenta de dificultades. El cuestionario de satisfacción fue respondido por 355 niños/as (90,8\%). En todas las preguntas cerradas más del $80 \%$ muestran satisfacción con la intervención y recomendarían su uso. Conclusiones. El marco de referencia de Orem permite diseñar intervenciones para cambio de conductas asociadas a alimentación. Su uso es factible en el contexto escolar, requiere de decisión y compromiso de los profesores. La estrategia puede ser incorporada al curriculum y contribuir al desarrollo de objetivos transversales. La satisfacción con la intervención fue alta.

Palabras clave: Nutrición escolar, sobrepeso, obesidad, autocuidado, conducta alimentaria.

Agradecimientos: Los autores desean expresar su agradecimiento a: La llustre Municipalidad de La Pintana, a los directores de los colegios, sus profesores, alumnos participantes y sus apoderados. A los estudiantes de la Escuela de Enfermería y de la Facultad de Educación quienes colaboraron en calidad de ayudantes. A Gabriela Bucarey, egresada del Programa de Magíster en Enfermería, por información aportada respecto al uso del software. A la Comisión Nacional de Investigación Científica y Tecnológica y su Fondo Nacional de Investigación y Desarrollo en Salud (FONIS) y la Pontificia Universidad Católica de Chile quienes hicieron posible la realización de este estudio.

\section{BIBLIOGRAFÍA}

1. Ministerio de Salud. Encuesta nacional de salud. ENS 2009-2010. pp 128-129. Chile [internet]. Disponible en: http://www.minsal.gob.cl/portal/url/item/bcb03d7bc28b64dfe040010165012d23.pdf

2. Ministerio de Salud. Obesidad en Chile, Un Problema de Salud Pública Chile; 2008.

3. Ministerio de Salud. DEIS. Diagnóstico nutricional integrado de la población menor de 6 años, por regiones, Diciembre 2007.

4. Crovetto $M$, Zamorano $F$, Medinelli $N$, Angelina $S$. Estado nutricional, conocimientos y conductas en escolares de kinder y primer año básico en 3 escuelas focalizadas por obesidad infantil en la comuna de valparaíso, chile. Rev. chil. nutr. [internet]. 2010 [citado 2012 Ago 22]; 37(3): 309-320. Disponible en: http:// www.scielo.cl/scielo.php?script=sci_arttext\&pid=S071775182010000300006\&/ng=es. doi: 10.4067/S071775182010000300006.

5. Larson NI, Neumark-Sztainer D, Hannan PJ, Story M. Family meals during adolescence are associated with higher diet quality and healthful meal patterns during young adulthood. J Am Diet Assoc 2007; 107: 1502-10.

6. El cerebro adolescente Los hábitos de salud para toda la vida se definen a los 16 años. [internet] disponible en: http://www.psicoadolescencia.com.ar/docs/notic1105.pdf

7. Conicyt. Proyecto FONIS 10/20016 Prevención del sobrepeso y la obesidad en escolares chilenos: una apuesta a la capacidad emprendedora de los niños. 2010.

8. Núcleo de Estudios Metropolitanos Instituto de Estudios Urbanos UC Informe Final Indicador Calidad de Vida Urbana (ICVU) Índice de Calidad de Vida Urbana. [internet[ disponible en:http://www.ieut.cl/wp-content/ uploads/2011/05/Informe-Final-ICVU.pdf

9. Orem D. Nursing: concepts of practice. 6 ed. Saint Louis: 
Mosby; 2001

10. Kain J, Uauy R, Leyton D, Cerda R, Olivares, S. y Vio F. Efectividad de una intervención en educación alimentaria y actividad física para prevenir obesidad en escolares de la ciudad de Casablanca, Chile (2003-2004) Rev Méd Chil [revista en la Internet].; 2008 136(1): 22-30. Disponible en: http://www.scielo.cl/scielo.php?script=sci_ arttext\&pid=S0034-98872008000100003\&/ng=es. doi: 10.4067/S0034-98872008000100003.

11. Vio F, Salinas J Promoción de Salud y Calidad de Vida en Chile: Una política con nuevos desafíos. Rev Chil Nutr [revista en la internet]. Disponible en: http:// www.scielo.cl/scielo.php?script=sci_arttext\&pid=S071775182006000300006\&lng=es. doi: 10.4067/S071775182006000300006 .

12. Troncoso C, Amaya JP. Factores sociales en las conductas alimentarias de estudiantes universitarios. Rev. chil. nutr. [revista en la Internet]. 2009 Dic [citado 2012 Sep 03]; 36(4): 1090-1097. Disponible en: http:// www.scielo.cl/scielo.php?script=sci_arttext\&pid=S071775182009000400005\&/ng=es. doi: 10.4067/S071775182009000400005 .

13. Foresight. Tackling Obesities: Future Choices - Project report. United Kingdom: Department of Innovation Universities and Skills. 2007; available form: http://www. bis. gov.uk/assets/foresight/docs/obesity/17.pdf

14. Moore JB, Pawloski L, Goldberg P, Kyeung M, Stoehr A, Baghi H. Childhood Obesity Study: A Pilot Study of the Effect of the Nutrition Education Program Color My Pyramid. J Nurs Sch 2009; 25; 3:230-9.

15. Moore JB, Gaffney K, Pawloski L, Jaimovich S, Campos C. Maternal-Child Nutrition Practices and Pediatric Overweight/Obesity in the United States and Chile: $A$ Comparative Study. J Pediatr Nursing 2011; 27 (5) 44-9.

16. Jaimovich S, Campos C, Campos MS, Moore J, Pawloski
L, Gaffney, K. Estado Nutricional y Conductas Asociadas a la Nutrición en Escolares. Rev Chil Pediatr 2010; 81 (6): 515-22.

17. Campos, C., Jaimovich, S., Moore, J. Pawloski, L., Gaffney, K., González, M. (2008). Operaciones de Autocuidado asociadas a la nutrición en madres de escolares chilenos. Rev Horizonte Enfermería. 19(2):45-53.

18. Romeo Cardone J. Los objetivos fundamentales transversales en busca de un curriculo holistico. Estud. Pedagóg. 2001. Disponible en <http://www.scielo.cl/scielo.php?script=sci_ arttext\&pid $=$ s0718-07052001000100009\&/ng=es\&nr m=iso>. Accedido en 31 agosto 2012. Doi: 10.4067/ s0718-07052001000100009.

19. DAEM Combarbalá Objetivos Fundamentales Transversales de la Educación General Básica 2011 disponible en http:// daemcombarbala.cl/objetivos-transversales-educacionchilen/

20. Labbé C, Matamala C, Astudillo G, Hinostroza JE. Uso de TIC por parte de profesores de Lenguaje, Matemática y Ciencias en educación media: Un estudio exploratorio disponible en www.ciie2012.cl/download.php?file=sesiones/266.pdf

21. Fahlman MM, Dake JA, McCaughtry N, Martin J. A pilot study to examine the effects of a nutrition intervention on nutrition knowledge, behaviors, and efficacy expectations in middle school children J Sch Health 2008; 78(4):216-22.

22. Hamre B.K, et al. Implementation fidelity of My Teaching Partner literacy and language activities: Association with preschoolers' language and literacy growth. Early Child Res Quart 2009 doi:10.1016/j.ecresq.2009.07.002.

23. Durlak J.A. The importance of doing well in whatever you do: A commentary on the special section, Implementation research in early childhood education. Early Child Res Quart 2010 doi:10.1016/j.ecresq.2010.03.003

24. Gobierno de Chile. Programa Elige Vivir Sano. 2011 disponible en http://www.eligevivirsano.cl/ 